nung Dermatologie, Gefäßchirurgie oder Angiologie erwerben das Zertifikat, wenn sie folgende Voraussetzungen erfüllen:

- Mitgliedschaft in der DGP

- Nachweis von 20 Fortbildungspunkten im phlebologischen Bereich je Jahr in den letzten 3 Jahren (z.B. Teilnahme an offiziellen Fortbildungsaktivitäten der DGP und / oder von der DGP offiziell anerkannten Fortbildungsveranstaltungen)

- Nachweis einer schwerpunktmäßigen phlebologischen Tätigkeit

- Ablegen eines Kolloquiums zu phlebologischen Inhalten
Ärzte ohne abgeschlossene Weiterbildung Phlebologie und ohne Facharztbezeichnung Dermatologie, Gefäßchirurgie oder Angiologie erwerben das Zertifikat, wenn sie folgende Voraussetzungen erfüllen:

- Mitgliedschaft in der DGP

- Nachweis von 20 Fortbildungspunkten im phlebologischen Bereich je Jahr in den letzten 3 Jahren (z.B. Teilnahme an offiziellen Fortbildungsaktivitäten der DGP und / oder von der DGP offiziell anerkannten Fortbildungsveranstaltungen)

- Nachweis einer schwerpunktmäßigen phlebologischen Tätigkeit Teilnahme am Grundkurs Phlebologie und min-

destens 2 von der DGP anerkannten Hands-on Workshops

- Ablegen eines Kolloquiums zu phlebologischen Inhalten

Das Zertifikat ist für 3 Jahre gültig. Es wird nach Vorlage von mindestens 60 Fortbildungspunkten im phlebologischen Bereich in den vorangegangenen 3 Jahren auf Antrag erneuert.

Die Unterlagen werden beim Sekretariat der DGP eingereicht. Die Bearbeitungsgebühr beträgt $150 €$ für den Erstantrag. Weitere Informationen finden Sie auf der Website der DGP www.phlebology.de

Dr. Erika Mendoza

\title{
Bringen Sie sich ein! Ehrenamt in der Phlebologie
}

Unsere Gesellschaft hat viele Aufgaben, im Wesentlichen die Unterstützung der täglichen Arbeit zur wissenschaftlichen Aufarbeitung unseres Fachbereichs, die Fortbildung und die Sicherung eines hohen Standards bei der Versorgung der Patienten. Somit sind wir alle täglich darin involviert.

Viele unserer Mitglieder beteiligen sich darüber hinaus an den verschiedenen Aufgaben unserer Fachgesellschaft. Nicht nur direkt im Vorstand und Beirat, sondern darüber hinaus in vielen anderen Bereichen: In der Schriftleitung der Zeitschrift Phlebologie, im wissenschaftlichen, in vielen
Arbeitsgemeinschaften, in Form von Fortbildungsveranstaltungen und - besonders arbeitsintensiv - in den Leitlinienkommissionen und in der Vorbereitung unserer gemeinsamen Jahrestagungen.

All diese Aktivitäten werden selbstverständlich ehrenamtlich ausgeführt, sprich ohne Honorar. Demgegenüber bekommt man durch sein Engagement andere Entschädigungen, wie das Kennenlernen von weiteren Interessierten und ähnlich gesinnten Menschen, das gemeinsame Erarbeiten von Projekten und dabei das Erweitern des eigenen Horizonts und nicht zuletzt die
Freude, zu sehen, wenn ein Projekt gut abgeschlossen wird, wenn wir Fortschritte machen. Schöne Beispiele hierzu sind die Freigabe aller Kosten rund um die Kompressionsstrümpfe Klasse I, auch in der An- und Ausziehhilfe für Pflegebedürftige oder die Erfolgsgeschichte unseres Fortbildungszertifikats Phlebologie.

Nicht nur die Zahl der Mitglieder ist erfreulicherweise gestiegen, sondern auch unsere Aktivitäten. Jederzeit sind wir auf Unterstützung durch unsere Mitglieder angewiesen. Sprechen Sie uns an - telefonisch, per Mail oder persönlich auf den Tagungen.

Dr. Erika Mendoza

\section{Brief an die Delegierten des 121. Deutschen Ärztetags:}

Beschluss der Bundesärztekammer senkt die Zahl von interdisziplinären Gefäßspezialisten für Venenkrankheiten! Die Zusatzweiterbildung Phlebologie darf nicht abgeschafft werden!

Sehr geehrte Damen und Herren,

die Deutsche Gesellschaft für Phlebologie protestiert scharf gegen die Pläne der Bundesärztekammer, die Zusatzweiterbildung Phlebologie abzuschaffen und empfindet 